\title{
Reduced miR-519d-3p levels in the synovium and synovial fluid facilitate the progression of post-traumatic osteoarthritis by targeting VEGF
}

\author{
JIANLONG GAO and SILONG XIA
}

\author{
Department of Orthopedics, The Affiliated Jianhu Hospital of Nantong University, Yancheng, Jiangsu 224700, P.R. China
}

Received March 26, 2021; Accepted July 26, 2021

DOI: $10.3892 /$ etm.2021.10913

\begin{abstract}
The present study aimed to investigate the expression and clinical significance of miR-519d-3p in patients with post-traumatic osteoarthritis (PTOA). The levels of miR-519d-3p in the synovium and synovial fluid (SF) of all subjects were detected by reverse transcription-quantitative polymerase chain reaction. The results of the present study demonstrated that the levels of miR-519d-3p in the synovium and SF of patients with PTOA were significantly lower, but that the VEGF content was significantly higher, compared with that of control group. Dual-luciferase reporter and Western blot assays demonstrated that VEGF was a target gene of miR-519d-3p. Furthermore, miR-519d-3p inhibitor-induced cell apoptosis, and cell cycle arrest could be partially reversed by silencing VEGF. Additionally, the level of miR-519d-3p in the synovium and SF of patients with PTOA was negatively correlated with the level of VEGF. ROC analysis demonstrated that miR-519d-3p levels in the synovium and SF could effectively differentiate patients with PTOA from healthy controls, with areas under the ROC curve of 0.928 and 0.896 , respectively. In conclusion, reduction of miR-519d-3p in the synovium and SF resulted in the upregulation of VEGF in patients with PTOA, and miR-519d-3p may be a potential therapeutic target of PTOA.
\end{abstract}

\section{Introduction}

Osteoarthritis (OA) is a type of degenerative arthropathy that commonly occurs in weight-bearing joints and is characterized by articular cartilage degeneration (1). Post-traumatic osteoarthritis (PTOA) is a complication of joint injury, accounting for $\sim 12 \%$ of $\mathrm{OA}$, in which the knee joint is one of the most commonly affected joints (2). Due to recent societal technological advancements, traffic accidents and sports injuries have increased (3). The incidence rate of PTOA is also

Correspondence to: Dr Jianlong Gao, Department of Orthopedics, The Affiliated Jianhu Hospital of Nantong University, 666 Nanhuan Road, Yancheng, Jiangsu 224700, P.R. China

E-mail: dgvc1109@163.com

Key words: miR-519d-3p, vascular endothelial growth factor, post-traumatic osteoarthritis, synovium, synovial fluid increasing (4). At present, the early diagnosis of PTOA is difficult (5). Due to a lack of specific interventions, PTOA seriously affects the daily life of patients (6). Therefore, investigation into potential biomarkers of PTOA will aid in improving joint function and the quality of life of patients with PTOA.

MicroRNAs (miRNAs) are important regulators of various diseases via interactions with the 3 '-untranslated regions (UTRs) of mRNAs (7). Increasing evidence has demonstrated the key roles that miRNAs serve in the progression of OA $(8,9)$. For example, the level of miR-16-5p is significantly increased in the chondrocytes of patients with OA compared with those of healthy controls (10). Additionally, miR-26a has been demonstrated to inhibit the activation of the NF- $\kappa \mathrm{B}$ signaling pathway, thereby alleviating synovial inflammation and cartilage injury in rats with OA (11). These findings suggested that miRNAs serve a critical role in cartilage homeostasis. In addition, previous studies have indicated an important role of miR-519 in the progression of various types of cancer, including breast cancer, gastric cancer and nasopharyngeal carcinoma (12-14). However, whether miR-519d-3p is dysregulated in patients with PTOA has not been previously reported.

In the present study, novel data are provided demonstrating that decreased levels of miR-519d-3p in the synovium and joint fluid promotes the progression of PTOA by inhibiting VEGF. The diagnostic values of miR-51d-3p and VEGF were further evaluated, which may shed light on the early prediction and intervention of PTOA.

\section{Materials and methods}

Patient samples. Between January 2018 and December 2019, 104 patients with PTOA were enrolled at the Affiliated Jianhu Hospital of Nantong University, with an average age of 52.30 \pm 9.97 (age range, 43-63 years; 55 males and 49 females) years. The inclusion criteria for patients with PTOA were as follows: i) all patients met the diagnostic criteria of PTOA according to clinical manifestations and imaging results and ii) all patients had at least one injured knee joint (left or right). The Kellgren-Lawrence (KL) scale (grades 0-4) was used to determine the severity: grade 0 , no radiographic features of OA are present; grade 1, possible narrowing of the joint space and osteophytic lipping; grade 2, definite osteophyte formation and possible narrowing of the joint space; grade 3, moderate osteophyte formation, definite narrowing of the joint space, 
some sclerosis and possible bone contour deformity; and grade 4, large osteophytes, marked narrowing of the joint space, severe sclerosis and definite bone contour deformity (15). Only knee OA patients with KL grades of $\geq 2$ were considered to be significant and were subsequently enrolled. If a patient had bilateral knee OA, the more affected knee was defined as the study knee. The X-ray was read by two orthopedic experts in our hospital in a blinded manner. KL scale classification was used to evaluate the imaging severity. The exclusion criteria were as follows: non-traumatic arthritis, rheumatoid arthritis, suppurative arthritis, gout, diabetes or knee mechanical injury. Synovial fluid (SF) was obtained from 106 knees of patients with PTOA at the time of knee arthroscopy or total knee replacement surgery. Human synovium was harvested from the knee joints of patients during total knee arthroplasty. The synovium and synovial tissue from knee joints donated voluntarily by 60 patients undergoing amputations $(51.40 \pm 10.69$ years; age range, 40-63 years; 33 males and 27 females) were obtained during the same period. There was no previous history of joint pain, joint activity restriction or joint swelling, no disease affecting the synthesis or metabolism of bone or joints. Synovitis and articular cartilage degeneration were excluded by the knee joint examination before, during and after surgery. There were no significant differences between the two groups regarding age, sex or other general information. The present study was approved by the Research Ethics Committee of the Affiliated Jianhu Hospital of Nantong University, and all the participants provided written informed consent to participate in this study.

Reverse transcription-quantitative polymerase chain reaction (RT-qPCR). SF was obtained from 106 knees of patients with PTOA at the time of knee arthroscopy or total knee replacement surgery. Human synovium was harvested during total knee arthroplasty from the knee joints of patients.

Next, $4 \mathrm{ml}$ joint fluid was centrifuged at $2,000 \mathrm{x} \mathrm{g}$ for $12 \mathrm{~min}$ at $4^{\circ} \mathrm{C}$. The supernatant was collected and stored at $-80^{\circ} \mathrm{C}$ for examination. Total RNA was isolated from the joint fluid samples $(4 \mathrm{ml})$ or the synovium using RNAVzol LS (Vigorous Biotechnology Beijing Co., Ltd.) according to the manufacturer's protocol. The concentrations and purities of the RNA samples were determined by measuring the optical density (OD) 260/OD280. RT-qPCR was performed using the $\operatorname{TaqMan}^{\mathrm{TM}}$ MicroRNA Reverse Transcription kit (Thermo Fisher Scientific, Inc.) The PCR amplifications were performed in a $12-\mu 1$ reaction system containing $6.0 \mu \mathrm{l}$ custom RT primer pool, $0.3 \mu \mathrm{l}$ dNTP, $3.0 \mu \mathrm{l}$ MultiScribe Reverse Transcriptase, $1.5 \mu 1$ 10X RT buffer, $0.19 \mu 1$ RNase Inhibitor, $1.01 \mu 1$ Nuclease-free water. The temperature protocol used for RT was as follows: $16^{\circ} \mathrm{C}$ for $30 \mathrm{~min}, 42^{\circ} \mathrm{C}$ for $30 \mathrm{~min}$ and $85^{\circ} \mathrm{C}$ for $5 \mathrm{~min}$, followed by storage at $4^{\circ} \mathrm{C}$. qPCR was performed using SYBR-Green Supermix (Bio-Rad Laboratories, Inc.). The PCR amplifications were performed in a $10 \mu \mathrm{l}$ reaction system containing $5 \mu \mathrm{l} \mathrm{SYBR-Green} \mathrm{Supermix,} 0.4 \mu \mathrm{l}$ forward primer, $0.4 \mu \mathrm{l}$ reverse primer, $2.2 \mu \mathrm{l}$ double distilled $\mathrm{H}_{2} \mathrm{O}$ and $2 \mu \mathrm{l}$ template cDNA. Thermocycling conditions were as follows: $95^{\circ} \mathrm{C}$ for $10 \mathrm{~min}$ followed by 40 cycles of $95^{\circ} \mathrm{C}$ for $15 \mathrm{sec}$ and $60^{\circ} \mathrm{C}$ for $1 \mathrm{~min}$. Relative miRNA expression was normalized to U6 using the $2^{-\Delta \Delta \mathrm{Cq}}$ method (16). The primers used were designed (17) and listed as follows: Universal stem-loop miR-545 reverse transcription primer, 5'-GAAAGAAGGCGAGGAGC
AGATCGAGGAAGAAGACGGAAGAATGTGCGTCTCGC CTTCTTTCAGTCATT-3'; U6 reverse transcription primer, 5'-CGCTTCACGAATTTGCGTGTCAA-3'; miR-545 PCR primer forward, 5'-GCTCAGTAAATGTTTATTAG-3' and reverse, 5'-CGAGGAAGAAGACGGAAGAAT-3'; U6 PCR primer forward, 5'-CTCGCTTCGGCAGCACATATACT-3' and reverse, 5'-CGCTTCACGAATTTGCGTGTCAT-3'.

Dual-luciferase reporter assay. The possible target gene of miR-519d-3p was predicted by TargetScan (http://www. targetscan.org/vert_72/). The 3'-UTR of VEGF was amplified from the genomic DNA of human primary chondrocytes using Platinum ${ }^{\mathrm{TM}}$ II Hot-Start Green PCR Master Mix (2X; Invitrogen; Thermo Fisher Scientific, Inc.). The PCR amplifications were performed in a $20 \mu \mathrm{l}$ reaction system containing $4 \mu \mathrm{l}$ 5X Platinum II PCR Buffer, $0.4 \mu \mathrm{l}$ forward primer, $0.4 \mu \mathrm{l}$ reverse primer, $0.4 \mu \mathrm{l}$ dNTP, $2.2 \mu \mathrm{l}$ double-distilled $\mathrm{H}_{2} \mathrm{O}$ and $2 \mu \mathrm{l}$ template cDNA, $0.32 \mu \mathrm{l}$ Platinum II Taq Hot-Start DNA Polymerase. Thermocycling conditions were as follows: Initial denaturation at $94^{\circ} \mathrm{C}$ for $2 \mathrm{~min}$, followed by 30 cycles of denaturation at $94^{\circ} \mathrm{C}$ for $15 \mathrm{sec}$, annealing at $60^{\circ} \mathrm{C}$ for $30 \mathrm{sec}$, extension at $68^{\circ} \mathrm{C}$ for $30 \mathrm{sec}$ and storage at $4^{\circ} \mathrm{C}$. The mutant was cloned using the Fast Mutagenesis System (TransGen Biotech). The PCR amplifications were performed in a $50 \mu 1$ reaction system containing $10 \mathrm{ng}$ genomic DNA $(2 \mu \mathrm{l}), 1 \mu \mathrm{l}$ forward primer, $1 \mu 1$ reverse primer, $25 \mu 12 \mathrm{X}$ TransStart FastPfu Fly PCR SuperMix, $21 \mu 1$ Nuclease-free water. Thermocycling conditions were as follows: Initial denaturation at $94^{\circ} \mathrm{C}$ for $2 \mathrm{~min}$, followed by 20 cycles of denaturation at $94^{\circ} \mathrm{C}$ for $20 \mathrm{sec}$, annealing at $55^{\circ} \mathrm{C}$ for $20 \mathrm{sec}$, extension at $72^{\circ} \mathrm{C}$ for $30 \mathrm{sec}$ and $4^{\circ} \mathrm{C}$ forever. Next, the amplified PCR fragment of VEGF-3'-UTR or VEGF-3'-UTR-Mut was cloned into the pmirGLO plasmid (Promega Corporation). The PCR products were ligated with EcoRI/XhoI-linearized pmiRGLO plasmid (Promega Corporation) to construct plasmids (pmirGLO-VEGF-3'-UTR, pmirGLO-VEGF-3'-UTR-Mut). All the expression plasmids were transformed into $E$. coli BL21(DE3) competent cells. The plasmid was isolated using GenElute ${ }^{\mathrm{TM}}$ Plasmid small quantity preparation kit (Thermo Fisher Scientific, Inc.). 293 cells were co-transfected with miR-519d-3p mimic and pmirGLO-VEGF-3'-UTR, pmirGLO-VEGF-3'-UTR-Mut using Vigofect transfection reagent (Vigorous Biotechnology Beijing Co., Ltd.), according to the manufacturer's protocol. 293 cells were seeded into 6 -well plates at a density of $10^{6}$ cells/well. In brief, $10 \mu \mathrm{l}$ Vigofect transfection reagent was mixed with $100 \mu \mathrm{l}$ serum-free culture. Meanwhile, $10 \mu 1 \mathrm{miR}-519 \mathrm{~d}-3 \mathrm{p}$ mimic and pmirGLO-VEGF-3'-UTR, pmirGLO-VEGF-3'-UTR-Mut was mixed with the aforementioned mixture. Next, the two mixtures were mixed and incubated at room temperature for $10 \mathrm{~min}$. Subsequently, the mixture was added to the 6 -well plate at a final concentration of $20 \mathrm{nM}$. Following transfection for $48 \mathrm{~h}$, the cells were collected for subsequent experiments. A dual-luciferase reporter assay was performed using a Dual-Luciferase Reporter Kit (Promega Corporation), according to the manufacturer's protocol (18). The relative luciferase activity was normalized to Renilla luciferase.

Enzyme-linked immunosorbent assay (ELISA). The levels of VEGF in the SF samples of all subjects were analyzed using a Human VEGF ELISA Kit (cat. no. ab222510; Abcam). 
Cell culture and cell transfection. Human primary chondrocytes (HC-a; cat. no. 4650) were purchased from ScienCell Research Laboratories. The cells were cultured in a chondrocyte medium (ScienCell Research Laboratories; cat. no. 4651), supplemented with $10 \%$ fetal bovine serum (FBS; Invitrogen; Thermo Fisher Scientific, Inc.), streptomycin $(100 \mathrm{mg} / \mathrm{ml})$ and penicillin $(100 \mathrm{U} / \mathrm{ml})$ at $37^{\circ} \mathrm{C}$ in a humidified atmosphere containing $5 \% \mathrm{CO}_{2}$. Only cells within the fifth passage were used for the subsequent experiments.

The siRNA, miR-519d-3p mimic, miR-519d-3p inhibitor and respective negative control (NC) was purchased from Shanghai GenePharma Co., Ltd. The sequences for siRNA, miR mimics and miR inhibitors were as follows: si-VEGF, 5'-GGCCGC CCAGGCUCCUG-3'; si-VEGF NC, 5'-CCGAUAGGUUUAC UGCCAAUU-3'; miR-519d-3p mimic, 5'-CCUCCAAAGGG AAGCGCUUUCUGUU-3'; miR-519d-3p mimic NC, 5'-UUC UCCGAACGUGUCACGU-3'; miR-519d-3p inhibitor, 5'-AAC AGAAAGCGCUUCCCUUUGGAGG-3'; miR-519d-3p inhibitor NC, 5'-CGAUAGGUUUACUGCCAAU-3'.

In brief, human primary chondrocytes cells were seeded into 6 -well plates at a density of $5 \times 10^{6}$ cells/well. Subsequently, the cells were transfected with the $20 \mathrm{nM}$ siRNA, $20 \mathrm{nM}$ miR-519d-3p mimic, 20 nM miR-519d-3p inhibitor or respective $20 \mathrm{nM}$ NC for $48 \mathrm{~h}$ using Hiperfect Transfection Reagent (Qiagen) according to the manufacturer's protocols. In brief, $12 \mu 1$ Hiperfect Transfection Reagent was mixed with $100 \mu \mathrm{l}$ serum-free chondrocyte medium (ScienCell Research Laboratories; cat. no. 4651). Additionally, $10 \mu 1$ siRNA, miR-519d-3p mimic, miR-519d-3p inhibitor or respective NC was mixed with serum-free chondrocyte medium. Next, the two mixtures were mixed and incubated at room temperature for $15 \mathrm{~min}$. Subsequently, the mixture was added to the 6 -well plate at a final concentration of $20 \mathrm{nM}$. Following transfection for $48 \mathrm{~h}$, the cells were collected for subsequent experiments.

Cell apoptosis assay. Flow cytometry was performed using a FITC/Annexin V Apoptosis Detection Kit I (BD Pharmingen ${ }^{\mathrm{TM}}$ ) according to the manufacturer's protocols. In brief, following transfection for $48 \mathrm{~h}$, cells were washed with PBS and resuspended in $100 \mu \mathrm{l}$ annexin binding buffer (1X), followed by incubation with $5 \mu$ l FITC Annexin V and $5 \mu \mathrm{l}$ PI working solution at room temperature for 15 min. Subsequently, $400 \mu \mathrm{l}$ annexin binding buffer (1X) was added to the mixture. Finally, the apoptosis rate of cells was analyzed by flow cytometry using a BD FACSCalibur system (LSRFortessa X-20; BD Biosciences). Data were analyzed using FCSalyzer v16.0.153 software (Huajun Software Park).

Cell cycle distribution assay. HC-a cells $\left(\sim 1 \times 10^{6}\right)$ were washed twice with PBS and were fixed in $70 \%$ ice-cold ethanol for $1 \mathrm{~h}$ at room temperature. The samples were centrifuged at $300 \mathrm{xg}$ for $5 \mathrm{~min}$ at $4^{\circ} \mathrm{C}$. The ethanol was removed, and the cells were exposed to $100 \mathrm{mg} / \mathrm{ml}$ R NaseA (Sigma-Aldrich; Merck KGaA) for $30 \mathrm{~min}$ at $37^{\circ} \mathrm{C}$. Cellular DNA was stained with propidium iodide (Nanjing KeyGen Biotech Co., Ltd.) for 15 min at room temperature. Cell cycle distributions were determined by flow cytometry using a BD FACSCalibur system (BD Biosciences), and data were analyzed using the ModFit software version 4.1 (Verity Software House, Inc.).
Table I. Demographic characteristics of patients with PTOA and HCs.

\begin{tabular}{lccc}
\hline Variable & PTOA $(\mathrm{n}=104)$ & $\mathrm{HC}(\mathrm{n}=60)$ & P-value \\
\hline Age, years & $52.30 \pm 9.67$ & $51.40 \pm 10.69$ & 0.21 \\
Sex, M/F & $55 / 49$ & $33 / 27$ & 0.37 \\
BMI, kg/m 2 & $25.30 \pm 1.50$ & $24.68 \pm 1.33$ & 0.43 \\
\hline
\end{tabular}

PTOA, post-traumatic osteoarthritis; HCs, healthy controls; BMI, body mass index.

Statistical analysis. Data were analyzed using SPSS software (version 20.0; IBM Corp.). The relevant data were expressed as the mean \pm standard deviation. A Mann-Whitney U test was used to examine differences between two groups. One-way analysis of variance, followed by the Tukey's post hoc test, was used to examine differences among multiple groups. Receiver operating characteristic (ROC) curves were created, and the areas under the curve (AUCs) were measured to further assess the specificity and sensitivity of miR-519d-3p as a diagnostic biomarker. Pearson's correlation was used to analyze the levels of miR-519d-3p and VEGF in the synovium and SF of patients with PTOA. $\mathrm{P}<0.05$ was considered to indicate a statistically significant difference.

\section{Results}

Demographic characteristics of patients with PTOA and healthy controls. To begin with, the demographic data of patients with PTOA and the healthy controls (HCs) were analyzed. As shown in Table I, there were no significant differences in age, sex or body mass index (BMI) between patients with PTOA and HCs. Furthermore, the differences among PTOA patients with K-L grade I, grade II, grade III and grade IV disease were compared. The results demonstrated that no significant differences in age, sex or BMI were identified among these groups (Table II).

Decreased level of miR-519d-3p in the synovium and $S F$ of patients with PTOA. Next, the levels of miR-519d-3p were analyzed in patients with PTOA and in HCs. Compared with the control group, the level of miR-519d-3p in the synovium and SF in the PTOA group was significantly decreased (Fig. 1A and B).

Decrease in miR-519d-3p in the synovium and SF of PTOA patients with increased $K$-L grades. The levels of miR-519d-3p were also compared among PTOA patients with different K-L grades. As demonstrated in Fig. 2A, the levels of miR-519d-3p in the synovium of patients in the grade III and IV groups were significantly lower than those in the grade I and II groups (Fig. 2A). By contrast, compared with the grade I group, the levels of miR-519d-3p in the SF of the grade II, III and IV groups were significantly lower (Fig. 2B).

VEGF is a target gene of miR-519d-3p. The target gene of miR-519d-3p was subsequently analyzed based on TargetScan (http://www.targetscan.org/vert_72/). A conserved binding 
Table II. Demographic characteristics of patients with PTOA with different KL grades.

\begin{tabular}{|c|c|c|c|c|c|}
\hline Variable & KL grade I $(n=22)$ & KL grade II $(n=28)$ & KL grade III $(n=29)$ & KL grade IV $(n=25)$ & P-value \\
\hline Age, years & $54.73 \pm 11.07$ & $51.57 \pm 10.33$ & $52.16 \pm 11.08$ & $51.60 \pm 7.69$ & 0.56 \\
\hline Sex, M/F & $11 / 11$ & $13 / 15$ & $14 / 15$ & $12 / 13$ & 0.47 \\
\hline $\mathrm{BMI}, \mathrm{kg} / \mathrm{m}^{2}$ & $25.52 \pm 2.02$ & $25.19 \pm 1.94$ & $25.27 \pm 1.96$ & $25.29 \pm 1.85$ & 0.58 \\
\hline
\end{tabular}

PTOA, post-traumatic osteoarthritis; KL, Kellgren-Lawrence; BMI, body mass index.
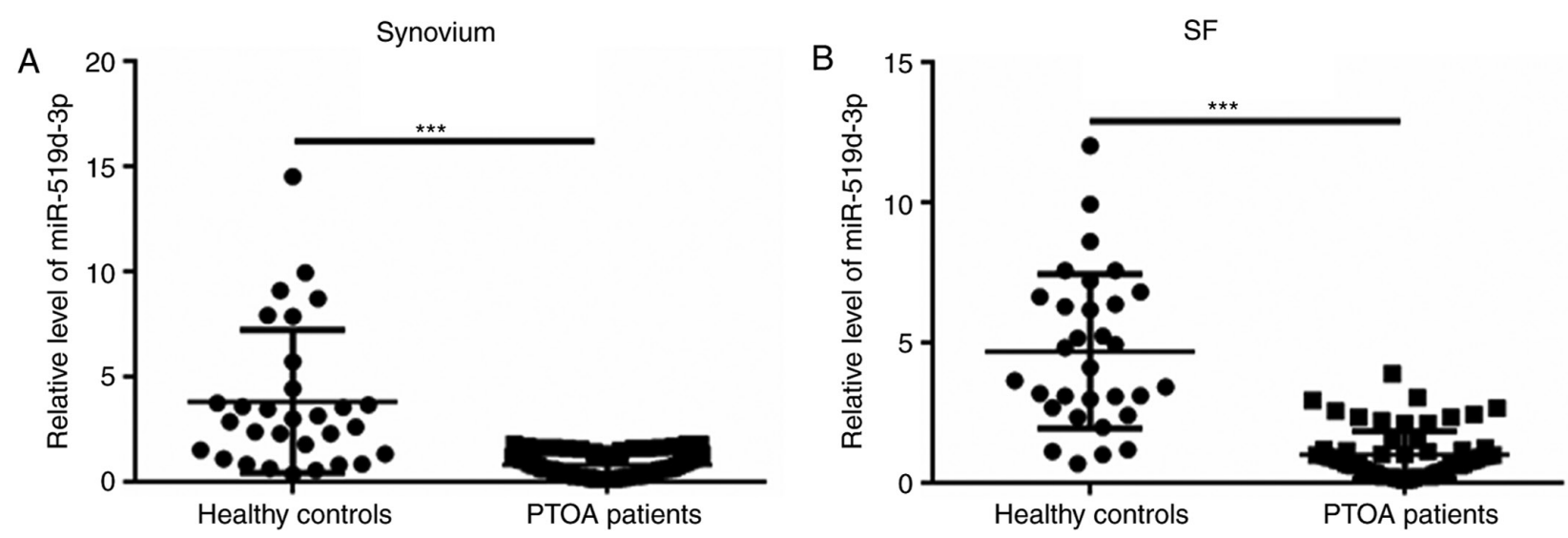

Figure 1. Reverse transcription-quantitative polymerase chain reaction analysis is performed to investigate the level of miR-519d-3p in patients with PTOA. The level of miR-519d-3p in (A) synovium and (B) SF of the PTOA group was significantly lower than that in healthy controls. ${ }^{* * *} \mathrm{P}<0.001$ vs. controls. SF, synovial fluid; PTOA, post-traumatic osteoarthritis.

A

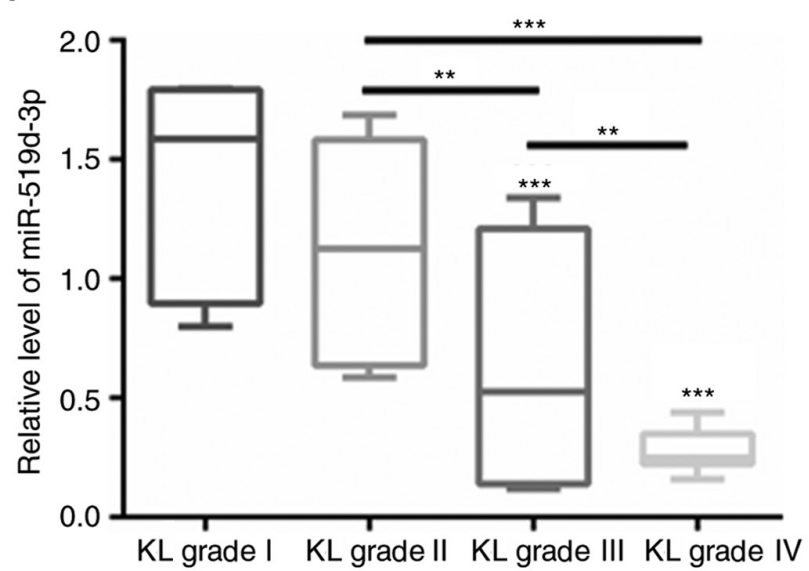

B

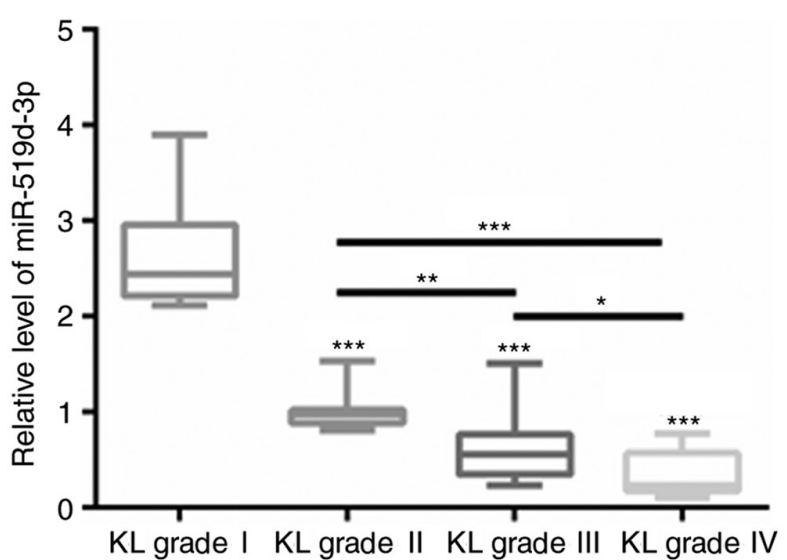

Figure 2. Reverse transcription-quantitative polymerase chain reaction is used to detect the expression of miR-519d-3p in the synovium and SF of PTOA patients with different KL grades. (A) The expression of miR-519d-3p in the synovium of patients in the grade II, III and IV groups was significantly lower than that in the grade I group. (B) Compared with the grade I group, the expression levels of miR-519d-3p in the SF of the grade II, III and grade IV groups were significantly decreased. ${ }^{*} \mathrm{P}<0.05,{ }^{* *} \mathrm{P}<0.01,{ }^{* * *} \mathrm{P}<0.001$ vs. healthy controls. SF, synovial fluid; PTOA, post-traumatic osteoarthritis.

site was identified in the 3'-UTR of enhanced VEGF (Fig. 3A), which has been suggested to be associated with OA progression (19). Dual-luciferase assays demonstrated that miR-519d-3p significantly suppressed the relative luciferase activity of pmirGLO-VEGR-3'-UTR, but no change in pmirGLO-VEGR-3'-UTR-Mut was observed (Fig. 3B). Next, miR-519d-3p mimic was transfected into human primary chondrocytes and RT-qPCR demonstrated that the level of miR-519d-3p was significantly increased following transfection with miR-519d-3p mimic, compared with NC (Fig. 3C), indicating successful transfection of miR-519d-3p mimic. Western blot analysis demonstrated that overexpression of miR-519d-3p significantly suppressed the expression of VEGF (Fig. 3D). By contrast, transfection with miR-519d-3p inhibitor significantly suppressed the level of miR-519d-3p compared with transfection with the NC (Fig. 3E). Meanwhile, transfection with an miR-519d-3p inhibitor significantly enhanced the expression of VEGF (Fig. 3F). Furthermore, the levels of VEGF were 
A

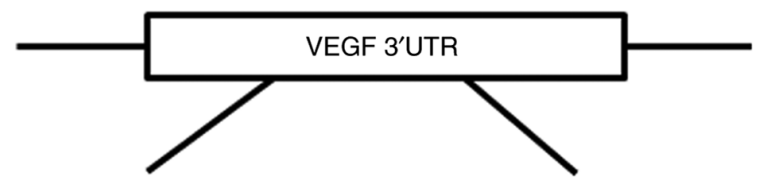

Position 193-199 of VEGF 3'UTR

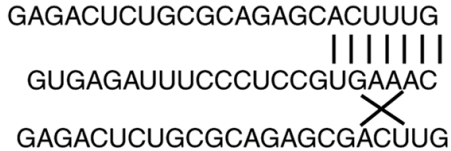

Mutant of VEGF $3^{\prime}$ UTR
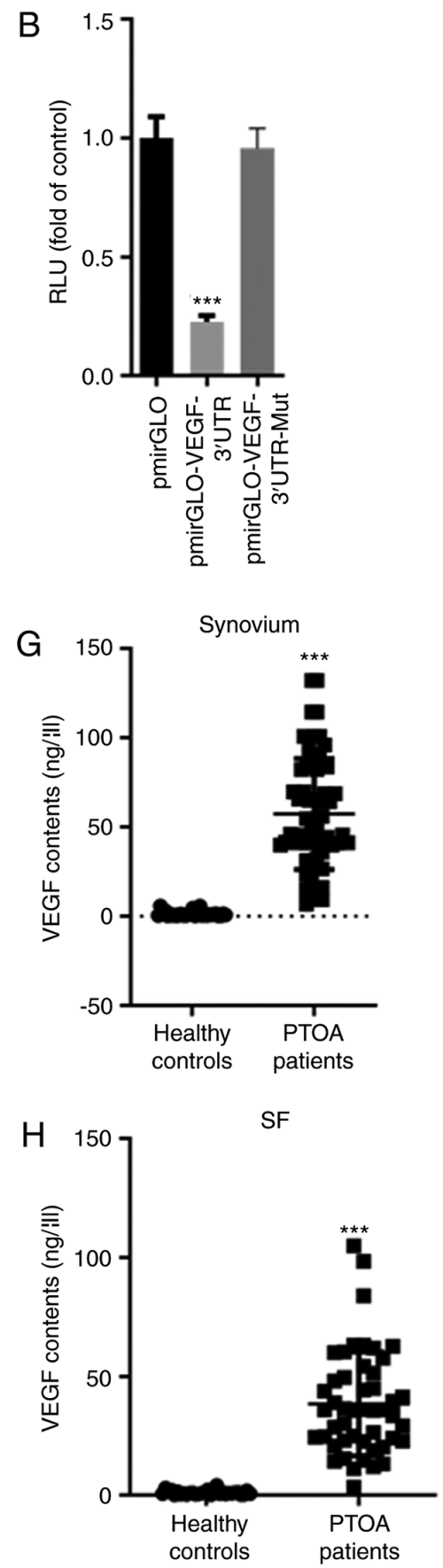

Figure 3. VEGF is a target gene of miR-519d-3p. (A) A conserved binding site was identified in the 3'-UTR of VEGF. (B) Dual-luciferase assay demonstrated that miR-519d-3p significantly suppressed the relative luciferase activity of pmirGLO-VEGR-3'-UTR. (C) Reverse transcription-quantitative polymerase chain reaction demonstrated that transfection with miR-519d-3p significantly increased the level of miR-519d-3p compared with transfection with the NC in primary human primary chondrocytes. (D) Western blot analysis demonstrated that overexpression of miR-519d-3p significantly suppressed the expression of VEGF. (E) Transfection with miR-519d-3p inhibitor significantly decreased the level of miR-519d-3p in primary human chondrocytes. (F) Inhibition of miR-519d-3p enhanced the expression of VEGF. The level of VEGF was revealed to be increased in (G) synovium and (H) SF of the PTOA group compared with the healthy controls. ${ }^{* * * *} \mathrm{P}<0.001$ vs. control. UTR, untranslated region; NC, negative control; SF, synovial fluid; PTOA, post-traumatic osteoarthritis; RLU, relative luciferase units.

revealed to be increased in the synovium and SF of the PTOA group, compared with that of the $\mathrm{HC}$ group (Fig. 3G and H).

miR-519d-3p inhibitor induces cell apoptosis, and cell cycle arrest is mediated via VEGF. Articular cartilage is a tissue that is nourished by $\mathrm{SF}(20)$. Increasing evidence has demonstrated an interaction between the SF microenvironment and chondrocytes and its role in cartilage degradation in OA $(21,22)$.
It is suggested that inflammatory synovial fluids of patients with reactive arthritis result in apoptosis and cell death of chondrocytes (21). Therefore, the effect of miR-519d-3p on the apoptosis and cell cycle arrest of chondrocytes was investigated. To further investigate whether miR-519d-3p is involved in the development of OA via VEGF, a specific siRNA targeting VEGF was selected. As demonstrated in Fig. 4A, transfection with si-VEGF significantly decreased the expression of 

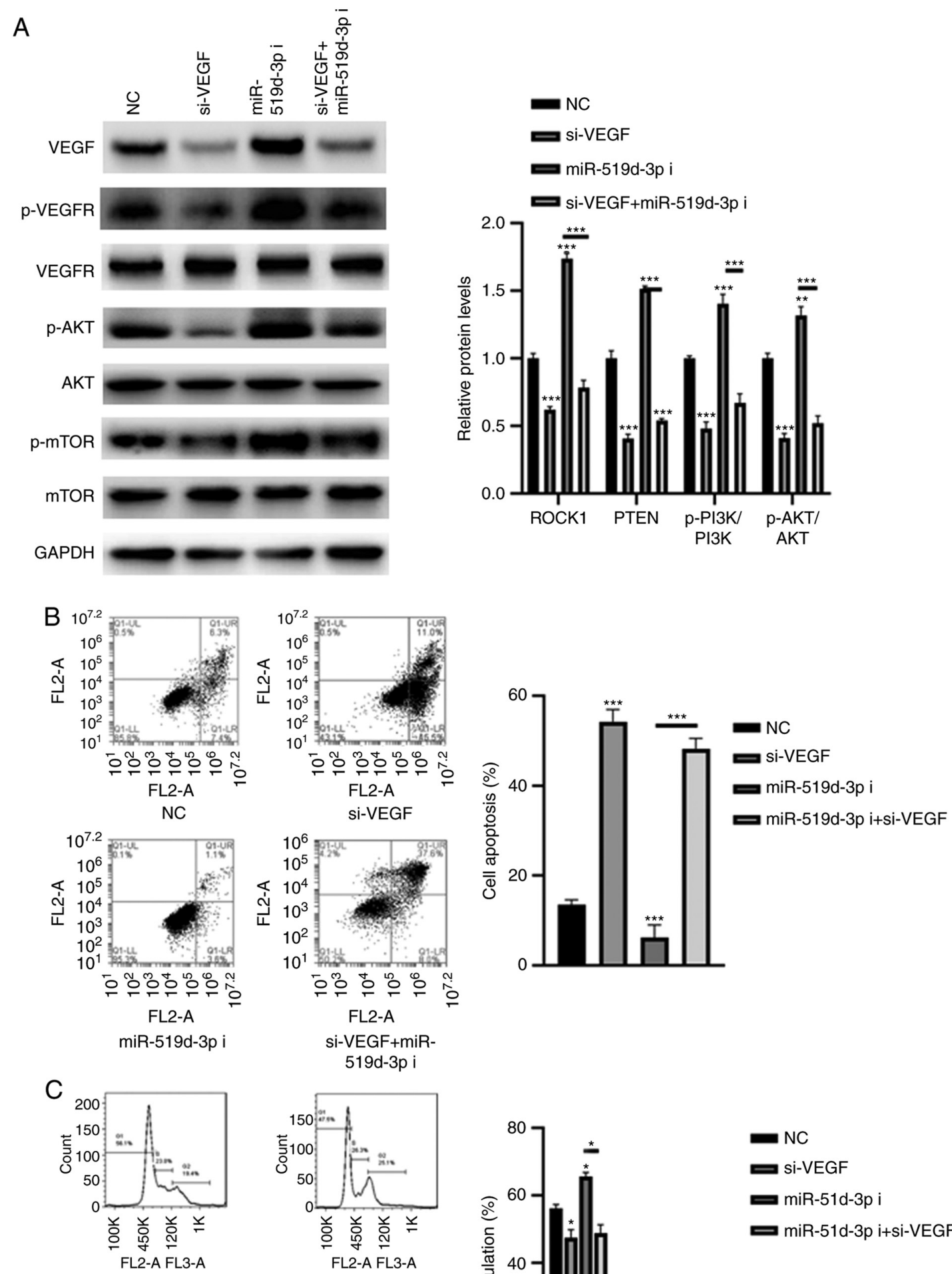

NC
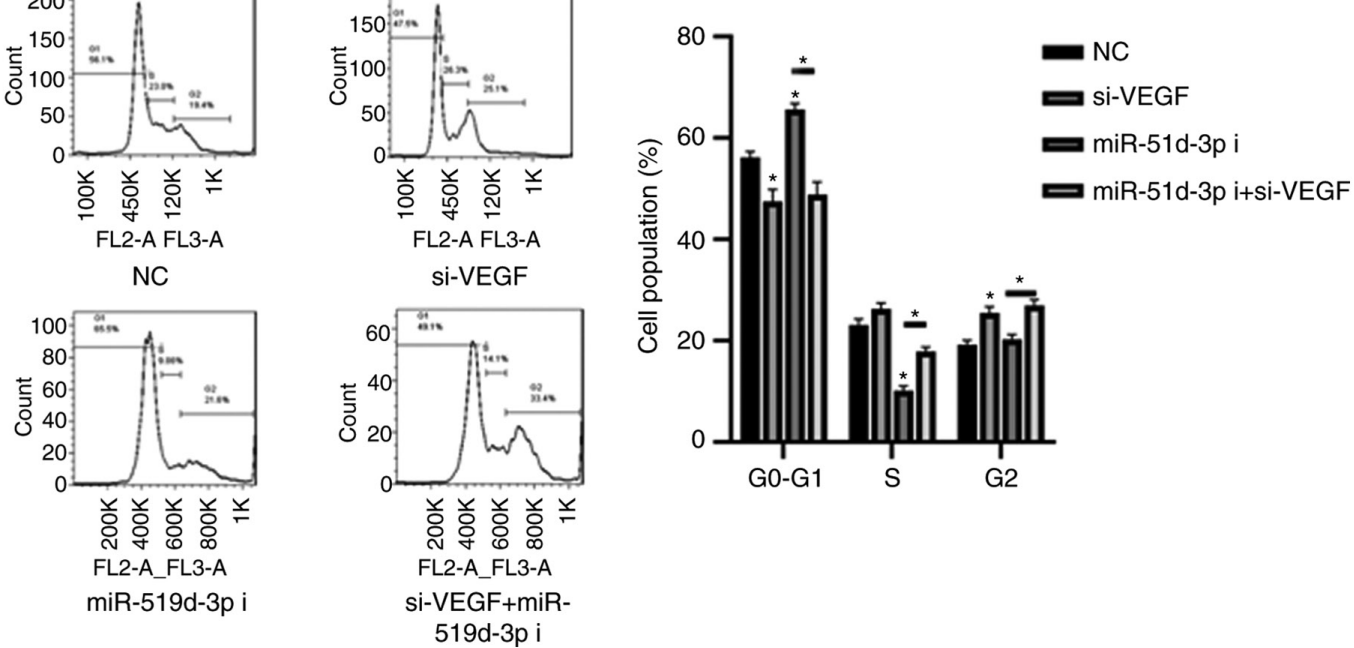

Figure 4. miR-519d-3p inhibitor induces cell apoptosis, and cell cycle arrest is mediated via VEGF. (A) Western blot analysis demonstrated that silencing VEGF significantly decreased the phosphorylation levels of VEGFR, AKT and mTOR, even in HC-a cells transfected with the miR-519d-3p inhibitor. (B) Flow cytometry demonstrated that inhibition of miR-519d-3p-induced cell apoptosis could be partially reversed by silencing VEGF. (C) miR-519d-3p inhibitor resulted in more cells at the $\mathrm{G} 0$ phase, but such effects could be abolished by knockdown of VEGF. ${ }^{*} \mathrm{P}<0.05,{ }^{* * * *} \mathrm{P}<0.001$ vs. as indicated. NC, negative control; HC-a, human primary chondrocytes. 

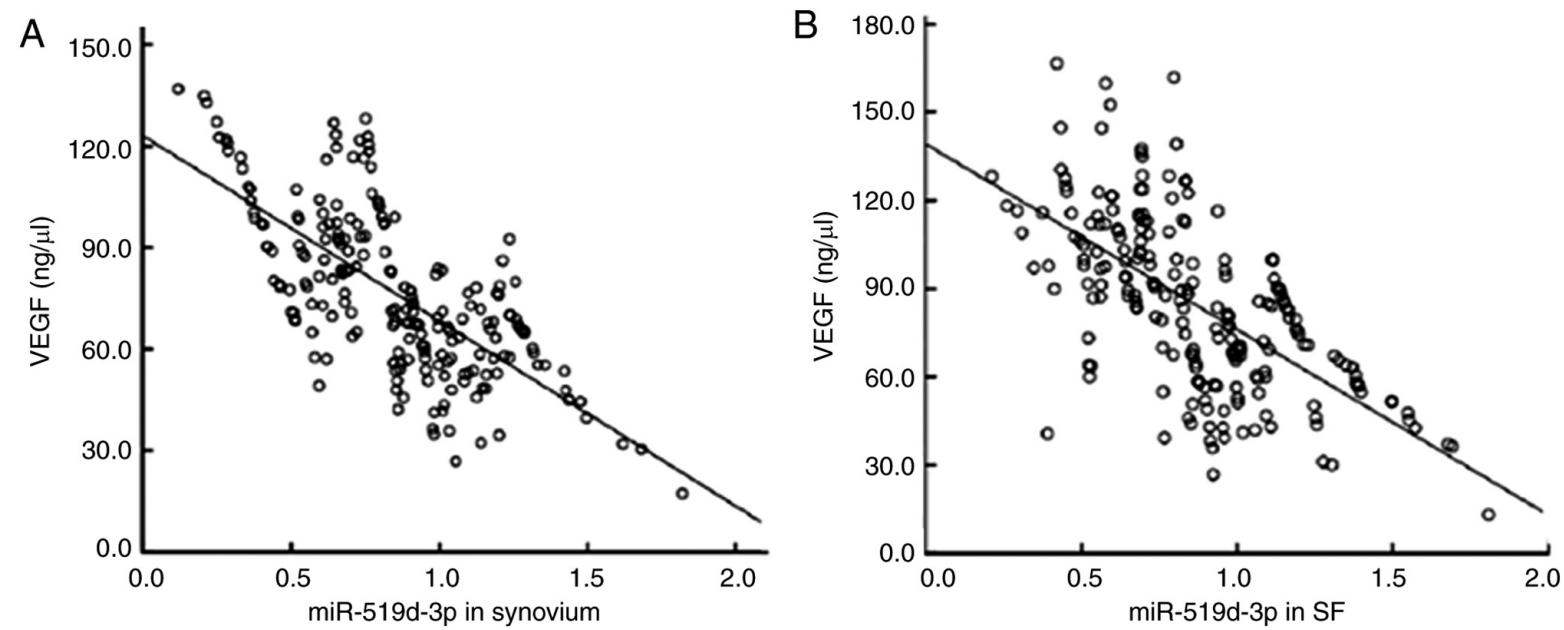

Figure 5. A negative correlation is identified between the expression of miR-519d-3p and VEGF in the synovium and SF of patients with PTOA. Pearson's correlation analysis demonstrated that the level of miR-519d-3p in the (A) synovium and (B) SF of patients with PTOA was negatively correlated with the VEGF content. SF, synovial fluid; PTOA, post-traumatic osteoarthritis.
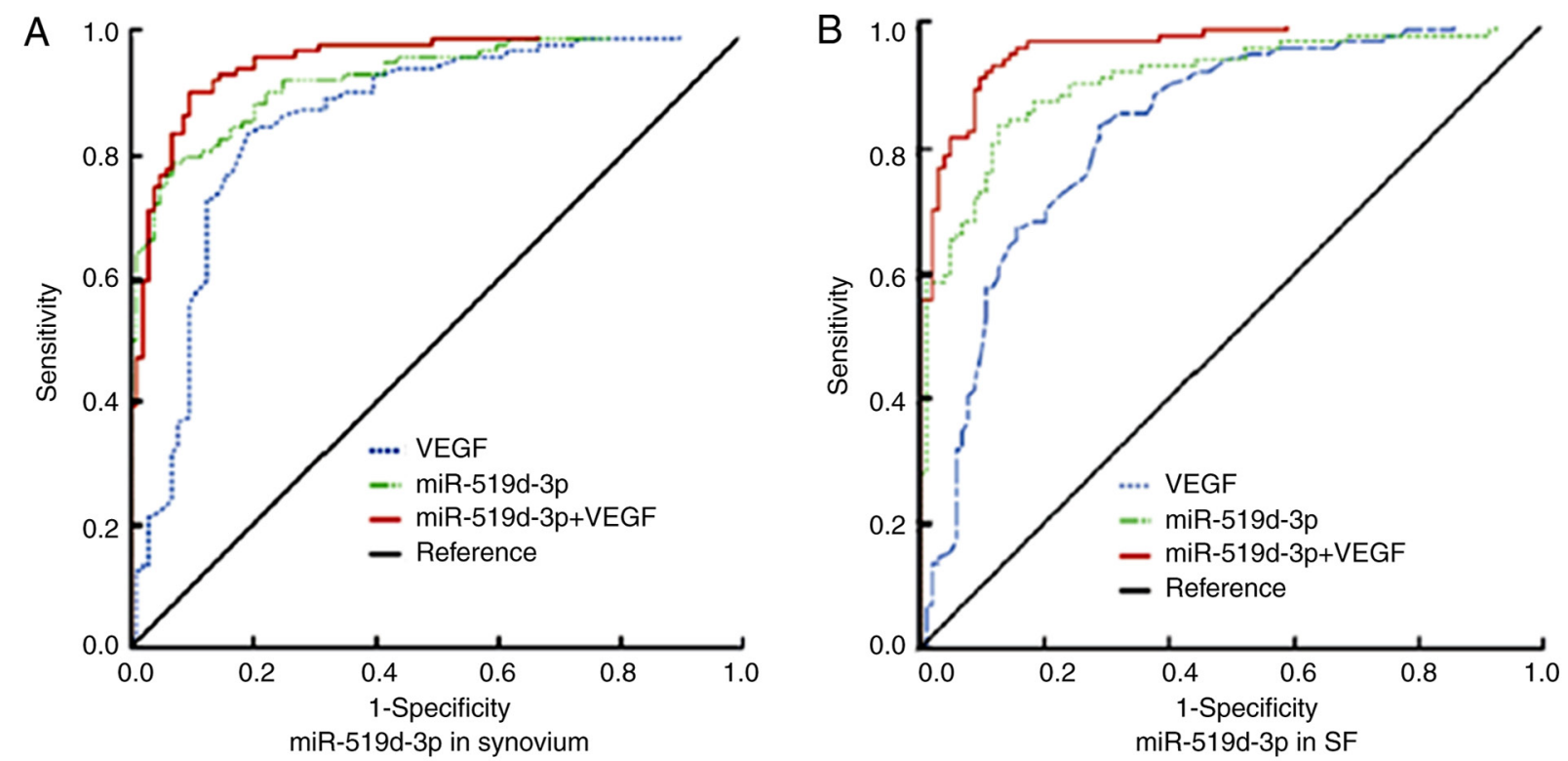

Figure 6. Receiver operating characteristic curve analysis was performed to evaluate the diagnostic value of miR-519d-3p in the synovium and SF of patients with PTOA. The diagnostic value of miR-519d-3p and VEGF mRNA in the (A) synovium and (B) SF was investigated in patients with PTOA. SF, synovial fluid; PTOA, post-traumatic osteoarthritis.

VEGF compared with the NC. Meanwhile, the silencing of VEGF significantly decreased the phosphorylation levels of VEGFR, AKT and mTOR, even in HC-a cells transfected with the miR-519d-3p inhibitor. Furthermore, the silencing of VEGF decreased HC-a cell apoptosis and induced cell division (Fig. 4B and C). By contrast, inhibition of miR-519d-3p resulted in more cell apoptosis and in the initiation of cell cycle arrest in G0 phase, compared with transfection with the NC (Fig. 4B and C). However, such effects could be reversed in HC-a cells transfected with si-VEGF (Fig. 4B and C).

Negative correlation between the level of miR-519d-3p and VEGF in the synovium and SF of patients with PTOA. Pearson's correlation analysis demonstrated that the levels of miR-519d-3p in the synovium and joint fluid of patients with
PTOA were negatively correlated with the contents of VEGF $(r=-0.701,-0.686, P<0.05$; Fig. 5A and B $)$.

Diagnostic value of miR-519d-3p and VEGF in the synovium and SF of patients with PTOA. The ROC curve analysis demonstrated that the AUC of VEGF in the synovium was 0.857 (95\% CI: 0.804-0.911). When the cut-off value was 0.855 , the sensitivity and specificity were 83.7 and $71.8 \%$, respectively (Fig. 5A). The AUC of miR-519d-3p in the diagnosis of PTOA was 0.928 (95\% CI: 0.894-0.962). When the cut-off value was 1.306 , the sensitivity and specificity were 78.8 and $93.3 \%$, respectively. The AUC of the combined use of VEGF and miR-519d-3p was 0.954 (95\% CI: 0.928-0.981), with a sensitivity and specificity of 90.4 and $90.4 \%$, respectively (Fig. 6A). 
By contrast, the AUC of miR-519d-3p in SF was 0.896 (95\% CI: 0.869-0.950). When the cut-off value was 1.492 , the sensitivity and specificity were 83.7 and $87.5 \%$, respectively (Fig. 6B). The AUC of VEGF was 0.830 (95\% CI: $0.774-0.886$ ). When the cutoff value was 0.785 , the sensitivity and specificity were 67.3 and $84.6 \%$, respectively. Furthermore, the combined AUC of the two was 0.963 (95\% CI: $0.941,0.986)$, with a sensitivity and specificity of 93.3 and $88.5 \%$, respectively (Fig. 6B). These observations suggested that miR-519d-3p in the synovium and SF may be a useful biomarker in the diagnosis of patients with PTOA.

\section{Discussion}

PTOA is mainly caused by trauma and is characterized by joint pain and biomechanical dysfunction (23). With the development of PTOA, disability develops and may seriously affect the quality of life of patients (1). At present, it remains difficult to diagnose and evaluate the disease in the early stages (24).

MiRNAs are suggested to be associated with the processes involved in OA pathogenesis, including cartilage homeostasis, extracellular matrix regulation, endochondral ossification, bone metabolism and angiogenesis $(25,26)$. In the present study, the levels of miR-519d-3p in the synovium and SF of patients with PTOA were significantly lower than those of the control group. Furthermore, the levels of miR-519d-3p in the synovium and SF of patients with PTOA decreased with an increasing KL grade. The results indicated that the downregulation of miR-519d-3p may contribute toward the development and progression of PTOA. Therefore, the upregulation of endogenous miR-519d-3p function could be an alternative therapeutic approach for PTOA prevention and treatment.

Abnormal expression of miR-519 has been widely reported in various tumor types $(14,27)$. For example, in several human carcinoma cell lines, including HeLa (cervical), HCT116 (colon), RKO (colon) and A2780 (ovarian), miR-519 has been shown to suppress cell proliferation by targeting HuR (27). In nasopharyngeal carcinoma cells, miR-519 suppresses cell proliferation by inhibiting URG4 (14). The present study provided novel data suggesting that VEGF is a target gene of miR-519d-3p. VEGF is a typical cytokine that may serve a key role in a variety of inflammatory pathways and angiogenesis (19). The overexpression of VEGF may cause long-term inflammatory effects and cartilage destruction of the synovium (28). In addition, VEGF may participate in rheumatoid arthritis, ankylosing spondylitis, OA and other inflammatory conditions (29). In support of these observations, the levels of VEGF in the synovium and SF of patients with PTOA were significantly higher than in the controls, suggesting that VEGF in the synovium and SF may participate in the occurrence and development of PTOA. Furthermore, miR-519d-3p was negatively associated with VEGF expression in the synovium and SF of patients with PTOA, suggesting the possible involvement of miR-519d-3p and VEGF in the pathogenesis of PTOA.

Apoptosis is a highly regulated cell death process that is extensively involved in the development, homeostasis and aging of various processes $(30,31)$. As the most common chronic joint disease in the elderly population, $\mathrm{OA}$ is characterized by the progressive destruction of articular cartilage $(32,33)$. Previous studies have indicated that chondrocyte apoptosis is correlated with the severity of OA $(34,35)$. In the present study, the effects of miR-519d-3p on chondrocyte apoptosis via VEGF were evaluated. The results demonstrated that silencing VEGF decreases chondrocyte apoptosis and induces cell division compared with the NC. By contrast, inhibition of miR-519d-3p induces HC-a cell apoptosis and cell cycle arrest. However, such effects could be eliminated by the knockdown of VEGF. These results suggested that the inhibition of miR-519d-3p induces chondrocyte apoptosis and cell cycle arrest by targeting VEGF.

To evaluate the diagnostic role of miR-519d-3p in PTOA, an ROC curve was constructed. The AUCs of miR-519d-3p in the synovium and SF were 0.954 and 0.963 , respectively, with sensitivities of 90.4 and $93.3 \%$, respectively. This suggests that miR-519d-3p and VEGF levels in the synovium and SF have diagnostic value for PTOA. However, there are certain limitations to the present study. To begin with, the sample size of this study was small, and the results need to be further confirmed with a larger sample size. Additionally, the present study did not investigate other possible target genes of miR-519d-3p that may also affect the pathogenesis of PTOA. Furthermore, whether the dose of miR-519d-3p under pathological conditions (reduction of $\sim 0.5$-fold) can inhibit the progression of PTOA has not been confirmed. In future studies, an animal model of PTOA will be established and administrate normal doses of miR-519d-3p mimic to elucidate this issue. Additionally, the present study investigated the decrease in miR-519d-3p in the apoptosis and cell cycle arrest of human primary chondrocytes. To further prove that the decrease in miR-519d-3p in synovial membrane and SF resulted in the upregulation of VEGF of patients with PTOA, it is necessary to investigate the effect of miR-519d-3p on synoviocytes and to evaluate its functional role in the SF microenvironment.

In conclusion, the present study demonstrated that the decrease in miR-519d-3p levels in the synovium and SF results in the upregulation of VEGF in patients with PTOA, and the combined use of miR-519d-3p and VEGF may improve the early diagnosis of PTOA.

\section{Acknowledgements}

Not applicable.

\section{Funding}

The present study was supported by a grant from the NANTONG supporting fund of The Affiliated Jianhu Hospital of Nantong University (NTJH-20190825).

\section{Availability of data and materials}

The datasets used and/or analyzed during the current study are available from the corresponding author on reasonable request.

\section{Authors' contributions}

JG performed the experiments, analyzed the data and wrote the manuscript. SX designed the experiments and analyzed the data. JG and SX confirmed the authenticity of all the raw data. All authors read and approved the final manuscript. 


\section{Ethics approval and consent to participate}

The present study was approved by the Research Ethics Committee of The Affiliated Jianhu Hospital of Nantong University (Nantong, China), and all the participants provided written informed consent for participation in this study.

\section{Patient consent for publication}

All patients provided written informed consent for the publication of data in this study.

\section{Competing interests}

The authors declare that they have no competing interests.

\section{References}

1. Takada S, Nakamura E, Sabanai K, Tsukamoto M, Otomo H, Kanoh S, Murai T, Fukuda H, Okada Y, Uchida S, et al: Attenuation of post-traumatic osteoarthritis after anterior cruciate ligament injury via inhibition of hedgehog signaling. J Orthop Res 38: 609-619, 2020.

2. Bodkin SG, Werner BC, Slater LV and Hart JM: Post-traumatic osteoarthritis diagnosed within 5 years following ACL reconstruction. Knee Surg Sports Traumatol Arthrosc 28: 790-796, 2020.

3. Brown SB, Hornyak JA, Jungels RR, Shah YY, Yarmola EG, Allen KD and Sharma B: Characterization of post-traumatic osteoarthritis in rats following anterior cruciate ligament rupture by non-invasive knee injury (NIKI). J Orthop Res 38: 356-367, 2020.

4. Cornelis FMF, de Roover A, Storms L, Hens A, Lories RJ and Monteagudo S: Increased susceptibility to develop spontaneous and post-traumatic osteoarthritis in Dot1l-deficient mice. Osteoarthritis Cartilage 27: 513-525, 2019.

5. Havryliuk H and Khimion L: Platelet autologous plasma in post-traumatic knee osteoarthritis treatment. J Clin Orthop Trauma 10: 42-45, 2019.

6. Rhon DI, Perez KG and Eskridge SL: Risk of post-traumatic knee osteoarthritis after knee injury in military service members. Musculoskelet Care 17: 113-119, 2019.

7. LaiZ and Cao Y: Plasma miR-200c-3p, miR-100-5p, and miR-1826 serve as potential diagnostic biomarkers for knee osteoarthritis: Randomized controlled trials. Medicine (Baltimore) 98: e18110, 2019.

8. Ma F, Li G, Yu Y, Xu J and Wu X: miR-33b-3p promotes chondrocyte proliferation and inhibits chondrocyte apoptosis and cartilage ECM degradation by targeting DNMT3A in osteoarthritis. Biochem Biophys Res Commun 519: 430-437, 2019.

9. Ma Y, Wu Y, Chen J, Huang K, Ji B, Chen Z, Wang Q, Ma J, Shen S and Zhang J: miR-10a-5p promotes chondrocyte apoptosis in osteoarthritis by targeting HOXA1. Mol Ther Nucleic Acids 14: 398-409, 2019.

10. Li L, Jia J, Liu X, Yang S, Ye S, Yang W and Zhang Y: MicroRNA-16-5p controls development of osteoarthritis by targeting SMAD3 in chondrocytes. Curr Pharm Des 21: 5160-5167, 2015.

11. Zhao Z, Dai XS, Wang ZY, Bao ZQ and Guan JZ: MicroRNA-26a reduces synovial inflammation and cartilage injury in osteoarthritis of knee joints through impairing the NF- $\kappa \mathrm{B}$ signaling pathway. Biosci Rep 39: 39, 2019.

12. Ren L, Li Y, Zhao Q, Fan L, Tan B, Zang A and Yang H: miR-519 regulates the proliferation of breast cancer cells via targeting human antigen R. Oncol Lett 19: 1567-1576, 2020.

13. Xu J, You Q, Wei Z, Fu H, Zhang Y, Hu Z and Cai Q: miR-519 inhibits epithelial-mesenchymal transition and biologic behavior of gastric cancer cells by down-regulating FOXQ1. Int J Clin Exp Pathol 13: 425-436, 2020 .

14. Yu G, Zhang T, Jing Y, Bao Q, Tang Q and Zhang Y: miR-519 suppresses nasopharyngeal carcinoma cell proliferation by targeting oncogene URG4/URGCP. Life Sci 175: 47-51, 2017.

15. Kellgren JH and Lawrence JS: Radiological assessment of rheumatoid arthritis. Ann Rheum Dis 16: 485-493, 1957.
16. Livak KJ and Schmittgen TD: Analysis of relative gene expression data using real-time quantitative PCR and the 2(-Delta Delta C(T)) method. Methods 25: 402-408, 2001.

17. Yang LH, Wang SL, Tang LL, Liu B, Ye WL, Wang LL, Wang ZY, Zhou MT and Chen BC: Universal stem-loop primer method for screening and quantification of microRNA. PLoS One 9: e115293, 2014.

18. Fang W, Guo J, Cao Y, Wang S, Pang C, Li M, Dou L, Man Y, Huang X, Shen T, et al: MicroRNA-20a-5p contributes to hepatic glycogen synthesis through targeting p63 to regulate p53 and PTEN expression. J Cell Mol Med 20: 1467-1480, 2016.

19. Hamilton JL, Nagao M, Levine BR, Chen D, Olsen BR and Im HJ: Targeting VEGF and its receptors for the treatment of osteoarthritis and associated Pain. J Bone Miner Res 31: 911-924, 2016.

20. Carballo CB, Coelho TR, de Holanda Afonso RC, Faria JC, Alves T, Monte SM, Ventura Matioszek GM, Moura-Neto V and Brito JM: Osteoarthritic synovial fluid and TGF- $\beta 1$ induce interleukin-18 in articular chondrocytes. Cartilage 11: 385-394, 2020.

21. Hoff P, Buttgereit F, Burmester GR, Jakstadt M, Gaber T, Andreas K, Matziolis G, Perka C and Röhner E: Osteoarthritis synovial fluid activates pro-inflammatory cytokines in primary human chondrocytes. Int Orthop 37: 145-151, 2013.

22. Pearson MJ, Herndler-Brandstetter D, Tariq MA, Nicholson TA, Philp AM, Smith HL, Davis ET, Jones SW and Lord JM: IL-6 secretion in osteoarthritis patients is mediated by chondrocyte-synovial fibroblast cross-talk and is enhanced by obesity. Sci Rep 7: 3451, 2017.

23. Singleton Q, Bapat S and Fulzele S: Post-traumatic osteoarthritis (PTOA) animal model to understand pathophysiology of osteoarthritis. Ann Transl Med 7 (Suppl 3): S81, 2019.

24. Zou YC, Li HH, Yang GG, Yin HD, Cai DZ and Liu G: Attenuated levels of ghrelin in synovial fluid is related to the disease severity of ankle post-traumatic osteoarthritis. Biofactors 45: 463-470, 2019.

25. van Meurs JB: Osteoarthritis year in review 2016: Genetics, genomics and epigenetics. Osteoarthritis Cartilage 25: 181-189, 2017.

26. Sondag GR, Mbimba TS, Moussa FM, Novak K, Yu B, Jaber FA, Abdelmagid SM, Geldenhuys WJ and Safadi FF: Osteoactivin inhibition of osteoclastogenesis is mediated through CD44-ERK signaling. Exp Mol Med 48: e257, 2016.

27. Abdelmohsen K, Srikantan S, Kuwano Y and Gorospe M: miR-519 reduces cell proliferation by lowering RNA-binding protein HuR levels. Proc Natl Acad Sci USA 105: 20297-20302, 2008.

28. Yuan Q, Sun L, Li JJ and An CH: Elevated VEGF levels contribute to the pathogenesis of osteoarthritis. BMC Musculoskelet Disord 15: 437, 2014

29. Nagao M, Hamilton JL, Kc R, Berendsen AD, Duan X, Cheong CW, Li X, Im HJ and Olsen BR: Vascular endothelial growth factor in cartilage development and osteoarthritis. Sci Rep 7: 13027, 2017.

30. Wang WT, Huang ZP, Sui S, Liu JH, Yu DM and Wang WB: microRNA-1236 promotes chondrocyte apoptosis in osteoarthritis via direct suppression of PIK3R3. Life Sci 253: 117694, 2020.

31. Feng L, Feng C, Wang CX, Xu DY, Chen JJ, Huang JF, Tan PL and Shen JM: Circulating microRNA let 7e is decreased in knee osteoarthritis, accompanied by elevated apoptosis and reduced autophagy. Int J Mol Med 45: 1464-1476, 2020.

32. Li GS, Cui L and Wang GD: miR-155-5p regulates macrophage M1 polarization and apoptosis in the synovial fluid of patients with knee osteoarthritis. Exp Ther Med 21: 68, 2021.

33. Li L, Lv G, Wang B and Kuang L: XIST/miR-376c-5p/OPN axis modulates the influence of proinflammatory M1 macrophages on osteoarthritis chondrocyte apoptosis. J Cell Physiol 235: 281-293, 2020.

34. Sun Y, Kang S, Pei S, Sang C and Huang Y: MiR93-5p inhibits chondrocyte apoptosis in osteoarthritis by targeting lncRNA CASC2. BMC Musculoskelet Disord 21: 26, 2020.

35. Tian J, Cheng C, Kuang SD, Su C, Zhao X, Xiong YL, Li YS and Gao SG: OPN Deficiency increases the severity of osteoarthritis associated with aberrant chondrocyte senescence and apoptosis and upregulates the expression of osteoarthritis-associated genes. Pain Res Manag 2020: 3428587, 2020. 